\title{
The Natural History of Patients with Prostate Specific Antigen Elevation Receiving Holmium Laser Enucleation of the Prostate: Prospective Cohort Study
}

\section{Hahn-Ey Lee}

Seoul National University College of Medicine

Hyun Sik Yoon

Seoul National University Hospital

ByungWon Kim

Kyungpook National University

Jungyo Suh

Seoul National University College of Medicine

Seung-June Oh ( $\nabla$ sjo@snu.ac.kr)

Seoul National University Hospital

\section{Research Article}

Keywords: Natural History, Patients, Holmium Laser, HoLEP

Posted Date: August 26th, 2021

DOI: https://doi.org/10.21203/rs.3.rs-820767/v1

License: (a) (i) This work is licensed under a Creative Commons Attribution 4.0 International License.

Read Full License 


\section{Abstract \\ Objectives}

To understand the natural history of patients with prostate specific antigen (PSA) elevation receiving Holmium Laser Enucleation of the Prostate (HoLEP) for benign prostatic hyperplasia (BPH), and to understand clinical factors correlated with PSA elevation.

\section{Subjects and Methods:}

Patients in the prospective database registry from our institution who underwent HoLEP between January 2010 and May 2020 were analyzed. The patients were classified into low $(<3.0 \mathrm{ng} / \mathrm{mL})$ and high ( $>=$ $3.0 \mathrm{ng} / \mathrm{mL}$ ) PSA groups at baseline. Intraoperative and early postoperative outcomes were evaluated. Postoperative evaluation was performed, with PSA being evaluated at the postoperative sixth month. Factors showing correlation with PSA elevation were analyzed.

\section{Results}

A total of 1297 patients with a mean age of $69.7( \pm 6.8$ SD) years were analyzed. The baseline PSA was $4.0( \pm 4.2) \mathrm{ng} / \mathrm{mL}$, with $713(55.0 \%)$ patients in the low PSA group $(1.6( \pm 0.8) \mathrm{ng} / \mathrm{mL})$, and $584(45.0 \%)$ patients in the high PSA group $(6.9( \pm 4.7) \mathrm{ng} / \mathrm{mL})$. The PSA level decreased postoperatively by $66.6( \pm$ $23.6) \%$ in all patients, and $54.3( \pm 23.9) \%$ in the low PSA group, and $79.6( \pm 14.7) \%$ in the high PSA group.

\section{Conclusions}

In patients with LUTS and elevated PSA, significant PSA reduction and symptom improvement was observed after HoLEP.

\section{Introduction}

Benign prostatic hyperplasia (BPH) is becoming increasingly common as elderly populations increase. As a result, increasingly more aging men presenting with lower urinary tract symptoms (LUTS) caused by $\mathrm{BPH}$ are seeking treatment [1]. Prostate specific antigen (PSA) elevation is often observed in patients with $\mathrm{BPH}$, which makes it sometimes difficult to differentiate from PSA elevation observed in prostate cancer (PCa) patients upon screening [2].

The management of patients with severe LUTS with persistently elevated or elevating PSA is a modern urologic dilemma [3]. However, men with continuously elevated PSA screening results and bothersome LUTS have not been able to receive objective explanations to the cause of PSA elevation. Therefore, it has been reported that such patients show increased cancer related worry, and may experience delay of 
optimal treatment [4]. So far, there have been strategic attempts to deal with populations with elevated PSA with negative prostate biopsies (P-Bx), and several algorithms have also been proposed [5-8], without clear consensus.

There is evidence reported which demonstrates the value of transurethral prostatectomy, including Holmium Laser Enucleation of the Prostate (HoLEP) in such patients with elevated PSA. Cho et al [9] evaluated the clinical significance of transurethral resection of the prostate in patients with BPH and an elevated PSA and reported a significant decrease in PSA and symptom improvement. However, understanding factors that could be related to PSA increase, how to interpret changes in PSA values still does not have a consensus, and the understanding is still not high. Although there have been a few studies analyzing the natural history of PSA, they have been mainly focused on cancer correlation [10]. Studies using prospective large cohort data are also limited. More detailed understanding of the natural history of PSA after prostatectomy for BPH and factors related to its elevation can help reduce unnecessary delay in treatment and may help establish appropriate guidelines for management of LUTS / BPH patients with elevated PSA.

We aimed to understand the natural history of patients with preoperative PSA elevation receiving HoLEP for BPH and to understand clinical factors correlated with PSA elevation.

\section{Subjects And Methods}

This study reviewed a prospective cohort of patients at our institution from January 2010 to May 2020, and who were enrolled in the prospective SNUH-BPH Database Registry. This study was previously approved by the Seoul National University Hospital Institutional Review Board (IRB No. H-0810-027-260) [11]. We obtained informed consent from all participants and/or their legal guardian(s),for data collection and analysis. The study protocol and contents associated with this study followed the Declaration of Helsinki guidelines.

The inclusion criteria included patients aged $\geq 50$ years who had a clinical diagnosis of BPH and underwent HoLEP. The exclusion criteria were patients who had a history of genitourinary cancer and pelvic surgery, and also neurogenic bladder dysfunction. The PSA (Roche Diagnostics, Indianapolis, IN, USA) measured most recently before HoLEP was used as the baseline and was classified into low (< $3.0 \mathrm{ng} / \mathrm{mL}$ ) and high ( $>=3.0 \mathrm{ng} / \mathrm{mL}$ ) PSA groups. All patients underwent urinalyses to detect urinary tract infections (UTI), which were treated if diagnosed, and followed up until urinalyses showed no evidence of infection. Medications for LUTS such as alpha blockers (AB), and 5-alpha reductase inhibitors (5-ARIs), number of acute urinary retention (AUR) episodes, international prostate symptom scores (IPSS) [12], total prostate volume (TPV) and transitional zone volume (TZV) measured by transrectal ultrasound, number of transrectal prostate biopsies (P-Bx), uroflowmetry (UFM) parameters, and urodynamic parameters including bladder outlet obstruction (BOO), and maximal detrusor pressure at Qmax (PdetQmax) were evaluated as previously described in literature [13]. 12-core biopsies were conducted near the base, mid-grand and apex until 2017. After 2018, 14-core P-Bx including both transitional zones 
were conducted [14]. HoLEP was conducted approximately 2 months after the P-Bx, therefore, there should have been no P-Bx impact on the preoperative PSA level.

HoLEP was conducted using the technique as we previously described in literature $[15,16]$. Enucleation was performed with the three-lobe techniques, and the enucleated prostatic adenoma was morcellated with a morcellator. The intraoperative parameters included operative time, energy use, weight of enucleated prostate tissue, morcellation time, and operative pathology. After surgery, all BPH medications were discontinued. If patients experienced OAB symptoms, anticholinergics, or beta 3 agonists were prescribed on-demand. With patients who were diagnosed with $\mathrm{PCa}$, the pathological results followed the Gleason staging system [17], and tumor volume percentage was recorded. These patients were referred to urologic oncologists and received treatment for PCa.

Postoperative parameters such as hospital stay, re-catherization, operative complications, and reoperation were collected. Complications were evaluated as previously described in literature [18]. Followup was performed according to the protocol at 2 weeks, 3 months, and 6 months postoperatively. IPSS and UFM were evaluated at each visit, and PSA levels at postoperative 6 months were measured. The natural history of patients were analyzed from baseline to intraoperative and postoperative follow-up. The PSA reduction after operation was analyzed, and work-up for patients with persistently elevated PSA were analyzed as well. When PSA levels were elevated at 6 months, they were continuously followed up every 4-6 months and if necessary, evaluated. For patients whose PSA levels normalized at 6 months, follow-up was discontinued. Urinalyses were conducted at postoperative 3 months and 6 months to identify UTIs and if detected, treated before PSA evaluation was conducted again.

We performed the statistical analyses using IBM SPSS Statistics version 22.0 (IBM, Armonk, NY, USA). Continuous variables were expressed as median and interquartile range (IQR), or mean and standard deviation (SD). We described categorical variables with the ratio of events (\%). We used the student $t$ test to analyze quantitative data. Binary variables were analyzed when a chi-square test was applicable, but the Fisher exact test was performed if the distribution of the equation deviated significantly from normal. All statistical analyses were two-sided, and the definition of statistical significance was a $p$ value of $\leq$ 0.05 .

The factors showing correlation with baseline PSA were analyzed using multivariable regression analysis. The distribution of PSA was right-skewed, so we determined to use the log transformation. Therefore, we first considered 14 variables, which were age of operation, symptom duration, preoperative $\mathrm{BPH}$ related operations, BOOI, AUR, alpha blockers, 5-ARIs, TPV, TZV, preoperative Qmax, baseline IPSS total score, PdetQmax, and baseline BMI as explanatory variables, and the final model was obtained by the stepwise variable selection procedure. Odds ratios (OR) with $95 \%$ confidence intervals (Cls) and corresponding $\mathrm{p}$ value were presented for simplification.

\section{Results}


The baseline characteristics of 1297 patients are shown in Table 1. The baseline PSA was $4.0( \pm 4.1$, SD) $\mathrm{ng} / \mathrm{mL}, 713(55.0 \%)$ were in the low PSA group $(1.6 \pm 0.8 \mathrm{ng} / \mathrm{mL})$, and $584(45.0 \%)$ patients were in the high PSA group $(6.9 \pm 4.7 \mathrm{ng} / \mathrm{mL})$. The average age of the patients was $69.7( \pm 6.8)$ years, the mean total IPSS score was $23.4( \pm 11.6)$, mean TPV was $68.3( \pm 33.6) \mathrm{mL}$, mean BOO index was $24.2( \pm 2.9) .294$ (22.7\%) patients experienced more than 1 AUR episode, and 442 (34.1\%) patients underwent more than one P-Bx. The characteristics of low and high PSA groups are also shown on Table 1. The BMI, postvoided residual, number of preoperative AURs, baseline PSA, TZV, the number of preoperative P-Bx, and BOO all were statistically significantly different between the low and high PSA groups $(p<0.05)$ 
Table 1

Baseline Patient Characteristics

\begin{tabular}{|c|c|c|c|c|}
\hline \multirow[t]{3}{*}{ Parameters (unit) } & \multirow[t]{3}{*}{$\begin{array}{l}\text { Whole population }(n= \\
\text { 1297) }\end{array}$} & \multirow{2}{*}{$\begin{array}{l}\text { Low PSA } \\
\text { group } \\
\text { PSA< } \\
3.0 \mathrm{ng} / \mathrm{mL}\end{array}$} & \multirow{2}{*}{$\begin{array}{l}\text { High PSA } \\
\text { group } \\
\text { PSA }= \\
3.0 \mathrm{ng} / \mathrm{mL}\end{array}$} & \multirow[t]{3}{*}{$\begin{array}{l}\mathrm{p}- \\
\text { value }\end{array}$} \\
\hline & & & & \\
\hline & & $(n=713)$ & $(n=584)$ & \\
\hline Age (year) & $69.7( \pm 6.8)$ & $69.5( \pm 6.6)$ & $69.8( \pm 7.2)$ & 0.554 \\
\hline Body mass Index (kg/m²) & $24.2( \pm 2.9)$ & $24.5( \pm 3.0)$ & $24.0( \pm 2.8)$ & 0.002 \\
\hline Symptom duration (months) & $42.5( \pm 64.6)$ & $43.9( \pm 72.9)$ & $40.7( \pm 52.8)$ & 0.373 \\
\hline \multicolumn{5}{|l|}{$\begin{array}{l}\text { Preoperative medications for } \\
\text { LUTS }\end{array}$} \\
\hline Alpha blockers, n (\%) & $924(71.2)$ & $373(28.8)$ & $923(71.2)$ & 1.00 \\
\hline $\begin{array}{l}\text { 5-alpha reductase inhibitors, } n \\
(\%)\end{array}$ & $447(34.5)$ & 849 (65.5) & $447(34.5)$ & 0.412 \\
\hline $\begin{array}{l}\text { International prostate } \\
\text { symptom score }\end{array}$ & $23.4( \pm 11.6)$ & $23.2( \pm 11.2)$ & $23.6( \pm 11.8)$ & 0.889 \\
\hline Maximum flow rate $(\mathrm{mL} / \mathrm{sec})$ & $9.3( \pm 4.7)$ & $9.6( \pm 4.7)$ & $9.2( \pm 4.7)$ & 0.440 \\
\hline $\begin{array}{l}\text { Post-voided residual volume } \\
(\mathrm{mL})\end{array}$ & $77.3( \pm 100.9)$ & $67.2( \pm 91.0)$ & $90.2( \pm 111.0)$ & $<.001$ \\
\hline $\begin{array}{l}\text { Acute urinary retention (times), } \\
\mathrm{n}(\%)\end{array}$ & & & & $<0.001$ \\
\hline 0 & $1003(77.3)$ & $600(84.2)$ & $403(69.0)$ & \\
\hline$>=1$ & $294(22.7)$ & $113(15.8)$ & $181(31.0)$ & \\
\hline 1 & $261(20.1)$ & $103(14.4)$ & $158(27.1)$ & \\
\hline 2 & $21(1.6)$ & $6(0.8)$ & $15(2.6)$ & \\
\hline 3 & $9(0.7)$ & $2(0.3)$ & $7(1.2)$ & \\
\hline 4 & $3(0.2)$ & $2(0.3)$ & $1(0.2)$ & \\
\hline $\begin{array}{l}\text { Prostate specific antigen } \\
(\mathrm{ng} / \mathrm{mL})\end{array}$ & $4.0( \pm 4.1)$ & $1.6( \pm 0.8)$ & $6.9( \pm 4.7)$ & $<0.001$ \\
\hline \multicolumn{5}{|l|}{ Prostate volume } \\
\hline Total prostate volume (mL) & $68.3( \pm 33.6)$ & $54.3( \pm 23.3)$ & $85.4( \pm 36.3)$ & $<.001$ \\
\hline
\end{tabular}

PSA, prostate specific antigen; LUTS, lower urinary tract symptoms; mean \pm SD 


\begin{tabular}{|c|c|c|c|c|}
\hline \multirow[t]{3}{*}{ Parameters (unit) } & \multirow[t]{3}{*}{$\begin{array}{l}\text { Whole population }(n= \\
\text { 1297) }\end{array}$} & $\begin{array}{l}\text { Low PSA } \\
\text { group }\end{array}$ & $\begin{array}{l}\text { High PSA } \\
\text { group }\end{array}$ & \multirow[t]{3}{*}{$\begin{array}{l}\text { p- } \\
\text { value }\end{array}$} \\
\hline & & $\begin{array}{l}\mathrm{PSA}< \\
3.0 \mathrm{ng} / \mathrm{mL}\end{array}$ & $\begin{array}{l}\mathrm{PSA}>= \\
3.0 \mathrm{ng} / \mathrm{mL}\end{array}$ & \\
\hline & & $(n=713)$ & $(n=584)$ & \\
\hline Transitional zone volume (mL) & $39.6( \pm 26.6)$ & $29.0( \pm 18.8)$ & $52.3( \pm 28.8)$ & $\dot{0}_{0.001}$ \\
\hline Prostate biopsies (times), n (\%) & & & & $\begin{array}{l}< \\
0.001\end{array}$ \\
\hline 0 & 855 (65.9) & $635(89.1)$ & $220(37.7)$ & \\
\hline$>=1$ & $442(34.1)$ & $113(15.8)$ & $364(62.3)$ & \\
\hline 1 & $357(27.5)$ & $70(9.8)$ & $287(49.1)$ & \\
\hline 2 & $57(4.4)$ & $6(0.8)$ & $51(8.7)$ & \\
\hline 3 & $21(1.6)$ & $1(0.1)$ & $20(3.4)$ & \\
\hline 4 & $6(0.5)$ & $1(0.1)$ & $5(0.9)$ & \\
\hline 5 & $1(0.1)$ & $0(0.0)$ & $1(0.2)$ & \\
\hline $\begin{array}{l}\text { Bladder outlet obstruction } \\
\text { index }\end{array}$ & $24.2( \pm 2.9)$ & $39.0( \pm 25.0)$ & $51.1( \pm 32.0)$ & $<.001$ \\
\hline $\begin{array}{l}\text { Maximal detrusor pressure at } \\
\text { Qmax }\end{array}$ & $67.1( \pm 27.5)$ & $60.8( \pm 25.2)$ & $74.9( \pm 28.1)$ & 0.002 \\
\hline
\end{tabular}

The perioperative parameters for all patients, and also according to low and high PSA groups are shown on Table 2. The mean values in the total patient group were; total operation time $59.8( \pm 31.1)$, and enucleation weight $25.2( \pm 27.1)$ grams. The mean hospital stay was $1.6( \pm 1.4)$ days. The morcellation time, enucleation time, enucleation weight, total operation time showed statistically significant differences between PSA low and high groups $(p<0.05)$. 
Table 2

Perioperative outcomes according to baseline PSA level

\begin{tabular}{|c|c|c|c|c|}
\hline Parameters & $\begin{array}{l}\text { Total population }(n= \\
\text { 1297) }\end{array}$ & $\begin{array}{l}\text { Low PSA } \\
\text { group } \\
\text { PSA< } \\
3.0 n g / m L \\
(n=713)\end{array}$ & $\begin{array}{l}\text { High PSA group } \\
\text { PSA }>= \\
3.0 n g / m L \\
(n=584)\end{array}$ & $\begin{array}{l}\mathrm{p}- \\
\text { value }\end{array}$ \\
\hline Total operation time (min) & $59.8( \pm 31.1)$ & $49.9( \pm 25.5)$ & $71.7( \pm 33.1)$ & $<.001$ \\
\hline Enucleation time (min) & $38.1( \pm 17.6)$ & $32.9( \pm 15.3)$ & $44.4( \pm 18.2)$ & $\begin{array}{l}<.001 \\
0 .\end{array}$ \\
\hline Morcellation time (min) & $10.0( \pm 8.2)$ & $7.6( \pm 6.0)$ & $12.6( \pm 9.4)$ & $<.001$ \\
\hline Enucleation weight (gm) & $25.2( \pm 27.1)$ & $16.1( \pm 14.9)$ & $36.3( \pm 33.6)$ & $<.001$ \\
\hline Hospital stay (days) & $1.6( \pm 1.4)$ & $1.5( \pm 1.2)$ & $1.6( \pm 1.5)$ & 0.213 \\
\hline Re-catheterization, n (\%) & $75(7.2)$ & $43(7.5)$ & $32(6.7)$ & 0.602 \\
\hline Clot evacuation, n (\%) & $45(4.3)$ & $22(3.9)$ & $23(4.9)$ & 0.438 \\
\hline Transfusion, n (\%) & $1(0.1)$ & $0(0.0)$ & $1(0.2)$ & 0.273 \\
\hline $\begin{array}{l}\text { Bladder neck contracture, } \mathrm{n} \\
(\%)\end{array}$ & $2(0.2)$ & $1(0.2)$ & $1(0.2)$ & 0.902 \\
\hline Urethral Stricture, n (\%) & $2(0.2)$ & $5(0.9)$ & $2(0.4)$ & 0.464 \\
\hline Reoperation rate, n (\%) & $2(0.2)$ & $1(0.2)$ & $1(0.2)$ & 1.0 \\
\hline
\end{tabular}

The postoperative changes in PSA, IPSS, and Qmax were analyzed (Table 3). The IPSS and Qmax improved significantly for two weeks, 3 month, and 6 month postoperative follow-up. The PSA level was $4.0( \pm 4.1) \mathrm{ng} / \mathrm{mL}$ at baseline and decreased to $1.0( \pm 0.9) \mathrm{ng} / \mathrm{mL}$ at postoperative 6 months. This decrease was similar in the low PSA group, which decreased from baseline $1.6( \pm 0.8)$ to $0.8( \pm 0.5)$ $\mathrm{ng} / \mathrm{mL}$, and in the high PSA group which decreased from baseline $6.9( \pm 4.7)$ to $1.2( \pm 1.1) \mathrm{ng} / \mathrm{mL}$ at 6 months postoperatively. These changes were all statistically significant when the 6 months value was compared to baseline $(p<0.005)$. 
Table 3

Postoperative changes

\begin{tabular}{|c|c|c|c|c|c|}
\hline & Baseline & $\begin{array}{l}\text { Postop } 2 \\
\text { weeks }\end{array}$ & $\begin{array}{l}\text { Postop } 3 \\
\text { months }\end{array}$ & $\begin{array}{l}\text { Postop } 6 \\
\text { months }\end{array}$ & $\begin{array}{l}\mathrm{p}- \\
\text { value }\end{array}$ \\
\hline IPSS (total) & $\begin{array}{l}23.4( \pm \\
11.6)\end{array}$ & $15.0( \pm 11.2)$ & $11.4( \pm 12.8)$ & $10.1( \pm 13.2)$ & $<.001$ \\
\hline $\begin{array}{l}\text { Maximum flow rate } \\
(\mathrm{mL} / \mathrm{sec})\end{array}$ & $9.3( \pm 4.7)$ & $20.0( \pm 9.7)$ & $21.5( \pm 10.9)$ & $22.0( \pm 13.2)$ & $\begin{array}{l}<.001 \\
0.001\end{array}$ \\
\hline Voided volume $(\mathrm{mL})$ & $\begin{array}{l}175.4( \pm \\
116.0)\end{array}$ & $\begin{array}{l}\text { 193.0 }( \pm \\
112.4)\end{array}$ & $\begin{array}{l}208.0( \pm \\
126.4)\end{array}$ & $\begin{array}{l}220.1( \pm \\
128.6)\end{array}$ & $\stackrel{<}{<.001}$ \\
\hline PVR (mL) & $\begin{array}{l}77.3( \pm \\
100.9)\end{array}$ & $26.3( \pm 38.8)$ & $18.8( \pm 32.3)$ & $18.1( \pm 33.3)$ & $\begin{array}{l}<.001 \\
0.001\end{array}$ \\
\hline PSA total (ng/mL) & $4.0( \pm 4.1)$ & - & - & $1.0( \pm 0.9)$ & $\begin{array}{l}<.001 \\
0.001\end{array}$ \\
\hline $\begin{array}{l}\text { Low PSA group } \\
\text { (Baseline PSA< } \\
\text { 3.0ng } / \mathrm{mL} \text { ) }\end{array}$ & $1.6( \pm 0.8)$ & - & - & $0.8( \pm 0.5)$ & \\
\hline $\begin{array}{l}\text { High PSA group } \\
\text { (Baseline PSA > = } \\
3.0 \mathrm{ng} / \mathrm{mL} \text { ) }\end{array}$ & $6.9( \pm 4.7)$ & - & - & $1.2( \pm 1.1)$ & \\
\hline
\end{tabular}

The natural history of PSA were analyzed. The PSA decrease from baseline to postoperative 6 months was shown in Fig. 1a. The decrease rate of PSA was 66.7 ( \pm 23.6$) \%$ in the total PSA group, and 54.3 ( \pm $23.9) \%$ in the low group, and $79.6( \pm 14.7) \%$ in the high group. The PSA decrease rate showed statistically significant correlation with the resected volume $(p<0.001)$. The natural history of PSA were analyzed (Fig. 1b). Preoperatively, 713 (55.0\%) patients were in the low PSA group, and 584 (45.0\%) patients were in the high PSA group. Among the baseline low PSA group patients $(n=713), 709$ (99.4\%) decreased and maintained low postoperatively, and among the baseline high PSA group patients $(\mathrm{n}=$ 584), 556 (95.2\%) were persistently postoperatively decreased.

In addition, the difference between high PSA and low PSA groups of preoperative PSA, relative to AUR, IPSS, TPV and BOOI, was analyzed using odds ratios. AUR was stratified according to groups with no AURs, and one or more episodes, IPSS stratified according to a cut-off level of 8 points [19], TPV stratified according to a cut-off level of $100 \mathrm{~mL}$ [20], and BOOI stratified with a cut-off of 40 [21]. The high PSA group showed an increased risk of AUR ( $>=1$ times) (odds ratio $(O R)=2.389,95 \%$ confidence interval (Cl) $[1.828,3.122], p<0.001)$, high TPV $(>=100 \mathrm{~mL})(\mathrm{OR}=8.368,95 \% \mathrm{Cl}[5.560,12.596], \mathrm{p}<0.001)$, and high $\mathrm{BOOI}(>=40)(\mathrm{OR}=2.389,95 \% \mathrm{Cl}[1.828,3.122], \mathrm{p}<0.001)$. Multiple regression showed that AUR, 
number of P-Bx, TPV, and BOOI showed statistically significant positive correlations, while 5-ARI medication history, IPSS, and BMI showed negative correlations with preoperative PSA at $5 \%$ significance level (Table 4).

Table 4

Characteristics Associated with PSA using univariable and multivariable regression analysis

\begin{tabular}{|lllll|}
\hline \multicolumn{5}{|c|}{ PSA } \\
\hline Parameters & Univariable analysis & & & \\
& Unadjusted OR $(95 \% \mathrm{Cl})$ & p-value & Adjusted OR $(95 \% \mathrm{Cl})$ & p-value \\
\hline AUR & $2.389(1.828,3.122)$ & $<0.001$ & $1.928(1.435,2.596)$ & $<0.001$ \\
\hline IPSS & $0.727(0.392,1.346)$ & 0.309 & $0.779(0.394,1.549)$ & 0.471 \\
\hline TPV & $8.368(5.560,12.596)$ & $<0.001$ & $7.166(4.734,11.208)$ & $<0.001$ \\
\hline BOOI & $2.396(1.912,3.000)$ & $<0.001$ & $2.030(1.591,2.593)$ & $<0.001$ \\
\hline $\begin{array}{l}\text { PSA, Prostate specific Antigen; Cl, Confidence Interval; AUR, Acute Urinary Retention; IPSS, } \\
\text { International Prostate Symptom Score; TPV, Total Prostate Volume; TZV, Transitional Zone Volume; } \\
\text { BOOI, Bladder Outlet Obstruction Index }\end{array}$ & & & \\
\hline
\end{tabular}

The surgical pathology after HoLEP was described. Adenocarcinoma was detected incidentally in 83 (6.4\%) of all patients, and 42 (5.9\%) patients were in the baseline low PSA group, and 41 (70\%) were in the baseline high PSA group $(p=0.426)$. The total Gleason score and tumor percentage were not statistically different between low and high PSA groups. Most of the patients had a tumor volume of $1 \%$ in both low and high PSA groups. 7 patients $(0.5 \%)$ were also diagnosed with other cancers (bladder TCC $(n=4,0.6 \%)$ prostate TCC $(n=3,0.4 \%))$, in addition to nodular hyperplasia and received follow-up treatment for cancer.

The characteristics of patients with elevated PSA 6 months postoperatively were evaluated. Among the 32 patients whose PSA was persistently elevated 6 months postoperatively, 4 (12.5\%) patients were from the low PSA group, and 28 (87.5\%) were from the high PSA group. In the patients whose PSA was persistently low at 6 months, 419 (33.1\%) patients had underwent more than one P-Bx at baseline, whereas in the patients whose PSA was persistently elevated at 6 months, 23 (71.9\%) patients had underwent more than one P-Bx at baseline.

The pathology of patients who underwent additional P-Bx after 6 month follow-up were also analyzed. Among the 4 patients who underwent postoperative $\mathrm{P}-\mathrm{Bx}$, one patient was diagnosed with prostatic adenocarcinoma.

\section{Discussion}


How to manage patients with elevated PSA is a topic that urologists face and experience difficulties with during clinical practice. Zackrisson et al [22] have reported that in patients with elevated PSA and one negative P-Bx, patients with larger prostates usually have BPH and not PCa. However, there are still no clear guidelines yet on how to manage such patients with persistently high or elevating PSA levels with negative P-Bx results. As a result, due to delay in appropriate treatment for bothersome LUTS, patients may experience a decrease in quality of life, and economic burden [23]. The ability to use serum PSA to predict a large prostate volume and decide treatment in BPH patients has been reported in literature [24]. Therefore, it is important to understand the natural history of patients with elevated PSA and factors related to the PSA elevation. Several strategies have been proposed in this regard. However, they do not safely eliminate the need for P-Bx [25].

Transurethral prostatectomy for BPH results in a significant diminution in PSA that correlates well with the weight of adenoma resected. The reduction in PSA shows correlation with the amount of adenoma removed. Patients with larger prostates have been reported in literature to show higher PSA levels before HoLEP and had a significantly greater decrease after surgery [26]. In a study following-up patients postoperatively, the investigators concluded that if pretreatment $\mathrm{P}-\mathrm{Bx}$ are negative and also operative specimens are benign in patients with high PSA values, in spite of PCa suspicion, those patients can be followed up like usual BPH patients [27]. Further long-term analysis may be helpful to better interpret these follow-up changes in PSA.

In our study, the patients who were diagnosed with PCa after transurethral prostatectomy showed similar incidence between the low and high PSA groups (5.9\% and 7.0\% respectively). Nunez et al [28] analyzed patients who were diagnosed with PCa despite a prior negative P-Bx, and suggested the limitations of PSA screening alone, and the benefits of having tissue for histopathological diagnosis during surgery. AlKhalil et al [29] have analyzed the interactions between BPH and PCa in large prostates. It has been reported that BPH arises in nearly all cases from the TZ, which leads to LUTS symptoms. There have been studies suggesting that patients with LUTS and elevated PSA have a lower incidence of PCa.

We analyzed the perioperative parameters of the patients in our study. The total operation time, enucleation time, morcellation time, enucleation weight were all statistically significantly higher in the preoperative high PSA group. This could also be correlated with the large prostate volume in the elevated PSA group patients. Trotsenko et al [30] have reported that postoperatively, the hospital stay was longer in the high PSA group, although the Clavien index was not statistically different between the high and low PSA groups in our study. There have been previous studies comparing the Clavien index between TPV $>=$ 100 and TPV $<100$ groups, with no clinically significant differences between the groups.

The potential correlation with BOO and PSA has been evaluated [31,32], with studies showing correlation between preoperative PSA levels in patients with $\mathrm{BOOI}>=40[9,33]$. High PSA levels in patients with LUTS are significantly associated with BOO, low PSA levels mean that definite BOO is unlikely [32]. Baten et al [34] have also reported that BOO can be the cause of chronic irritation of the prostate. This can be caused by high detrusor pressure at maximal voiding (PdetQmax). This may induce PSA to be released to 
the blood stream from the prostate. Also, BOO closely correlates with TPV [35], which in turn, closely correlates with PSA level.

It has been reported in literature that an elevated PSA is associated with increased risk of AUR [31, 33]. The most common cause of AUR is BPH, which includes increases in the amount of TZ prostate stroma and an increase in alpha 1 a receptors, which when stimulated, result in smooth muscle contraction. In $\mathrm{BPH}$, the TZ surrounding the urethra compresses it, and causes BOO [36]. The findings in our study are aligned with such previous studies in that the elevated PSA has shown correlation with AUR, BOO, and prostate volume [21].

The fact that 5-ARIs are negatively correlated with PSA is well explained in literature [37]. In our study, we were able to demonstrate that the decrease in PSA postoperatively was consistent with patients not receiving 5-ARI treatment, which is consistent with previously reported studies [34]. Stoner et al [38] studied that prostate volume returns to pretreatment levels within 12 weeks of 5-ARI discontinuation. Considering that our final PSA measurement was conducted 6 months postoperatively, and that 5-ARIs were discontinued postoperatively, it is unlikely that 5-ARI would have affected the PSA value. In order to have a better understanding of the impact of 5-ARI in PSA and patient outcome, further studies analyzing the correlation between HoLEP intraoperative parameters in high PSA patients are necessary.

Our study has the advantages of a large prospective cohort with data collected by a single experienced surgeon, biomarkers, imaging, urodynamic data, symptom scores, and follow-up schedule were all protocolized, which adds to the strength and value of this study. However, this study does have some limitations. First, that we could not analyze patients who did not receive surgical treatment and compare with those who underwent surgery was a limitation. In addition, the postoperative follow-up duration was not long, as follow-up was usually discontinued at 6 months if there were no abnormalities revealed. Also, there were also patients who could not receive P-Bx prior to prostatectomy due to comorbidities, so their PSA elevation may not have been properly evaluated. Patients who had elevated PSA, who however refused to receive surgery were not analyzed either. Another point was that we did not use the risk calculator. Finally, we did not use MRI fusion biopsies for PCa risk calculation in this study. Lamb et al [39] have reported that the sensitivity and specificity of MRI is not yet sufficient to replace TRUS biopsies. MRI fusion biopsies have become increasingly common in the other countries such as the US. However, in Korea, such biopsies have only recently become reimbursed [40]. Our data was collected before MRI fusion techniques were commonly used. Therefore, if we could analyze and compare patients with elevated PSA not receiving surgery, and monitor patients for a longer-term postoperatively, as well as analyze fusion MRI biopsy data in the future, we may obtain more valuable information helpful for clinical practice.

\section{Conclusion}

In patients with LUTS and elevated PSA, significant PSA reduction and symptom improvement was observed after HoLEP. In such patients, timely surgical intervention could be considered without any delay 
due to PSA follow-up. Understanding the natural history of patients with elevated PSA before and after HoLEP could help provide information which can be further utilized in guidelines for patient diagnosis and monitoring of PSA in the future.

\section{Declarations}

\section{Acknowledgments}

We are obliged to Ms.Yu Kyung Lee for valuable help in data acquisition.

\section{Author Contributions}

Conceptualization: Seung-June Oh.

Data creation: Hyun Sik Yoon, Jungyo Suh

Formal analysis: Hahn-Ey Lee, ByungWon Kim, Jungyo Suh

Investigation: Hahn-Ey Lee, Seung-June Oh.

Methodology: Hahn-Ey Lee, Seung-June Oh.

Supervision: Seung-June Oh.

Writing \pm original draft: Hahn-Ey Lee.

Writing \pm review \& editing: Hahn-Ey Lee, Seung-June Oh.

\section{References}

1. Roehrborn, C. G. The utility of serum prostatic-specific antigen in the management of men with benign prostatic hyperplasia. Int J Impot Res, 20 (Suppl 3), 19-26 (2008).

2. Nath, C. K. et al. Prostate-Specific Antigen Density: A Measurement to Differentiate Benign Hypertrophy of Prostate from Prostate Carcinoma. J Lab Physicians, 12 (1), 44-48 (2020).

3. Puppo, P. Repeated negative prostate biopsies with persistently elevated or rising PSA: a modern urologic dilemma. Eur Urol, 52 (3), 639-641 (2007).

4. Katz, D. A. et al. Health perceptions in patients who undergo screening and workup for prostate cancer. Urology, 69 (2), 215-220 (2007).

5. van Renterghem, K. et al. A new algorithm in patients with elevated and/or rising prostate-specific antigen level, minor lower urinary tract symptoms, and negative multisite prostate biopsies. Int Urol Nephrol, 42 (1), 29-38 (2010).

6. Choo, M. S. et al. Development of Decision Support Formulas for the Prediction of Bladder Outlet Obstruction and Prostatic Surgery in Patients With Lower Urinary Tract Symptom/Benign Prostatic 
Hyperplasia: Part I, Development of the Formula and its Internal Validation. Int Neurourol J, 21 (Suppl 1), 55-65 (2017).

7. Choo, M. S. et al. Development of Decision Support Formulas for the Prediction of Bladder Outlet Obstruction and Prostatic Surgery in Patients With Lower Urinary Tract Symptom/Benign Prostatic Hyperplasia: Part II, External Validation and Usability Testing of a Smartphone App. Int Neurourol J, 21 (Suppl 1), 66-75 (2017).

8. Kim, M. et al. Factors influencing nonabsolute indications for surgery in patients with lower urinary tract symptoms suggestive of benign prostatic hyperplasia: analysis using causal bayesian networks. Int Neurourol J, 18 (4), 198-205 (2014).

9. Cho, H. J. et al. The role of transurethral resection of the prostate for patients with an elevated prostate-specific antigen. Prostate Int, 2 (4), 196-202 (2014).

10. Ito, K. et al. Natural history of PSA increase with and without prostate cancer. Urology, 62 (1), 64-69 (2003).

11. Yuk, H. D. \& Oh, S. J. Perioperative Safety and Efficacy of Holmium Laser Enucleation of the Prostate in Patients Receiving Antithrombotic Therapy: A Prospective Cohort Study. Sci Rep, 10 (1), 5308 (2020).

12. Elkhodair, S., Parmar, H. V. \& Vanwaeyenbergh, J. The role of the IPSS (International Prostate Symptoms Score) in predicting acute retention of urine in patients undergoing major joint arthroplasty. Surgeon, 3 (2), 63-65 (2005).

13. Kang, M. et al. Urodynamic Features and Significant Predictors of Bladder Outlet Obstruction in Patients With Lower Urinary Tract Symptoms/Benign Prostatic Hyperplasia and Small Prostate Volume. Urology, 89, 96-102 (2016).

14. Choo, M. S. et al. Predictors of positive surgical margins and their location in Korean men undergoing radical prostatectomy. Int J Urol, 21 (9), 894-898 (2014).

15. Oh, S. J. Current surgical techniques of enucleation in holmium laser enucleation of the prostate. Investig Clin Urol, 60 (5), 333-342 (2019).

16. Zidan, H. E. et al. Diagnostic and prognostic value of serum miR-15a and miR-16-1 expression among egyptian patients with prostate cancer. IUBMB Life, 70 (5), 437-444 (2018).

17. Kim, M. et al. Clinical and pathologic characteristics of familial prostate cancer in Asian population., 80 (1), 57-64 (2020).

18. Piao, S. et al. Holmium Laser Enucleation of the Prostate is Safe for Patients Above 80 Years: A Prospective Study. Int Neurourol J, 20 (2), 143-150 (2016).

19. Porreca, A. et al. Preoperative multiparametric prostate magnetic resonance imaging: a safe clinical practice to reduce incidental prostate cancer in Holmium laser enucleation of the prostate. Cent European J Urol, 72 (2), 106-112 (2019).

20. Kuntz, R. M., Lehrich, K. \& Ahyai, S. A. Holmium laser enucleation of the prostate versus open prostatectomy for prostates greater than 100 grams: 5-year follow-up results of a randomised clinical trial. Eur Urol, 53 (1), 160-166 (2008). 
21. Kang, M. Y., Ku, J. H. \& Oh, S. J. Non-invasive parameters predicting bladder outlet obstruction in Korean men with lower urinary tract symptoms. J Korean Med Sci, 25 (2), 272-275 (2010).

22. Zackrisson, B. et al. Follow-up of men with elevated prostate-specific antigen and one set of benign biopsies at prostate cancer screening. Eur Urol, 43 (4), 327-332 (2003).

23. Ilic, D. et al. Prostate cancer screening with prostate-specific antigen (PSA) test: a systematic review and meta-analysis., 362, 3519 (2018).

24. Chung, B. H. et al. Relationship between serum prostate-specific antigen and prostate volume in Korean men with benign prostatic hyperplasia: a multicentre study. BJU Int, 97 (4), 742-746 (2006).

25. Borer, J. G. et al. Age specific prostate specific antigen reference ranges: population specific. J Urol, 159 (2), 444-448 (1998).

26. Romero-Otero, J. et al. Critical analysis of a multicentric experience with holmium laser enucleation of the prostate for benign prostatic hyperplasia: outcomes and complications of 10 years of routine clinical practice. BJU Int, 126 (1), 177-182 (2020).

27. Ozden, C. et al. Detection of prostate cancer and changes in prostate-specific antigen (PSA) six months after surgery for benign prostatic hyperplasia in patients with elevated PSA. Urol Int, 71 (2), 150-153 (2003).

28. Nunez, R. et al. Incidental prostate cancer revisited: early outcomes after holmium laser enucleation of the prostate. Int J Urol, 18 (7), 543-547 (2011).

29. Al-Khalil, S. et al. Interactions between benign prostatic hyperplasia (BPH) and prostate cancer in large prostates: a retrospective data review. Int Urol Nephrol, 48 (1), 91-97 (2016).

30. Trotsenko, P. et al. Efficacy, safety, and perioperative outcomes of holmium laser enucleation of the prostate-a comparison of patients with lower urinary tract symptoms and urinary retention.Lasers Med Sci, 2020.

31. van Renterghem, K. et al. Clinical relevance of transurethral resection of the prostate in "asymptomatic" patients with an elevated prostate-specific antigen level. Eur Urol, 52 (3), 819-826 (2007).

32. Laniado, M. E. et al. Serum prostate-specific antigen to predict the presence of bladder outlet obstruction in men with urinary symptoms. BJU Int, 94 (9), 1283-1286 (2004).

33. van Renterghem, K. et al. Long-term clinical outcome of diagnostic transurethral resection of the prostate in patients with elevated prostate-specific antigen level and minor lower urinary tract symptoms. Urol Int, 83 (1), 60-65 (2009).

34. Abedali, Z. A. et al. The Role of Prostate Specific Antigen Monitoring after Holmium Laser Enucleation of the Prostate. J Urol, 203 (2), 304-310 (2020).

35. Wadie, B. S. How correlated is BOO with different objective parameters commonly used in evaluation of BPH: a prospective study (Int Urol Nephrol, 2020).

36. Cahn, D. B. et al. Predicting Acute Urinary Retention in Patients with Elevated Post-Void Residuals. Curr Urol, 8 (2), 79-83 (2015). 
37. Shigemura, K. et al. Effect of Preoperative Dutasteride on Holmium Laser Enucleation of the Prostate. Urol Int, 104 (5-6), 356-360 (2020).

38. Stoner, E. The clinical effects of a 5 alpha-reductase inhibitor, finasteride, on benign prostatic hyperplasia. The Finasteride Study Group. J Urol, 147 (5), 1298-1302 (1992).

39. Lamb, B. W. et al. Is Prebiopsy MRI Good Enough to Avoid Prostate Biopsy? A Cohort Study Over a 1Year Period. Clin Genitourin Cancer, 13 (6), 512-517 (2015).

40. Ryoo, H. et al. Detection of prostate cancer using prostate imaging reporting and data system score and prostate-specific antigen density in biopsy-naive and prior biopsy-negative patients. Prostate Int, $8(3), 125-129$ (2020).

\section{Figures}


A.

\section{Baseline Low PSA Group Postoperative PSA Change at 6 Months}

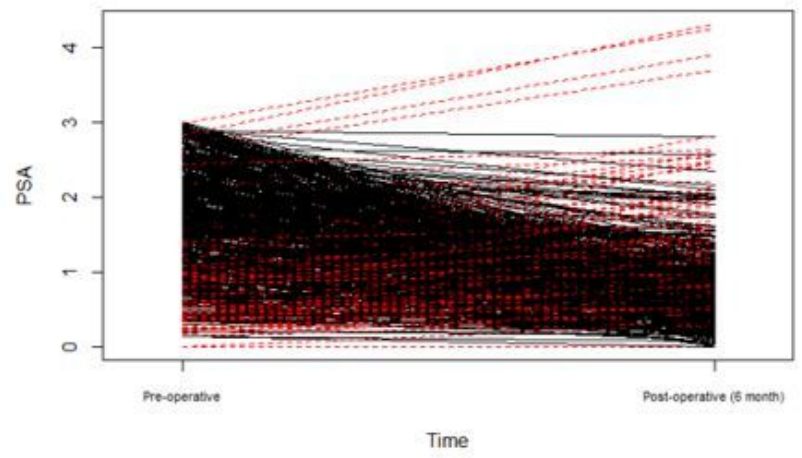

\section{Baseline High PSA Group Postoperative PSA Change at 6 Months}

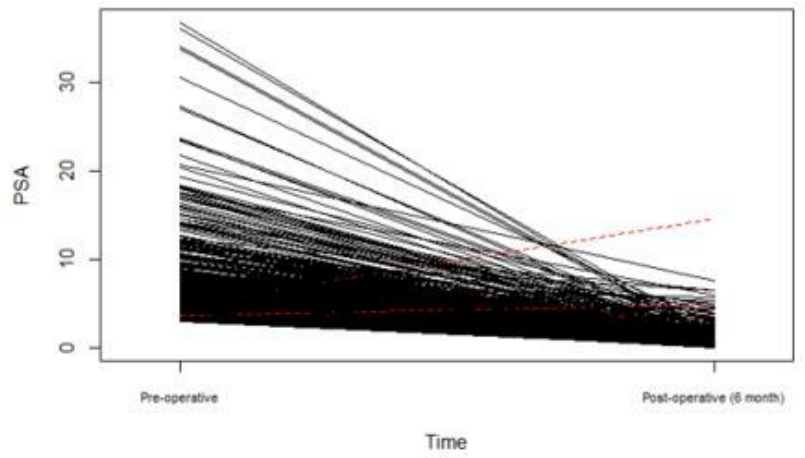

B.

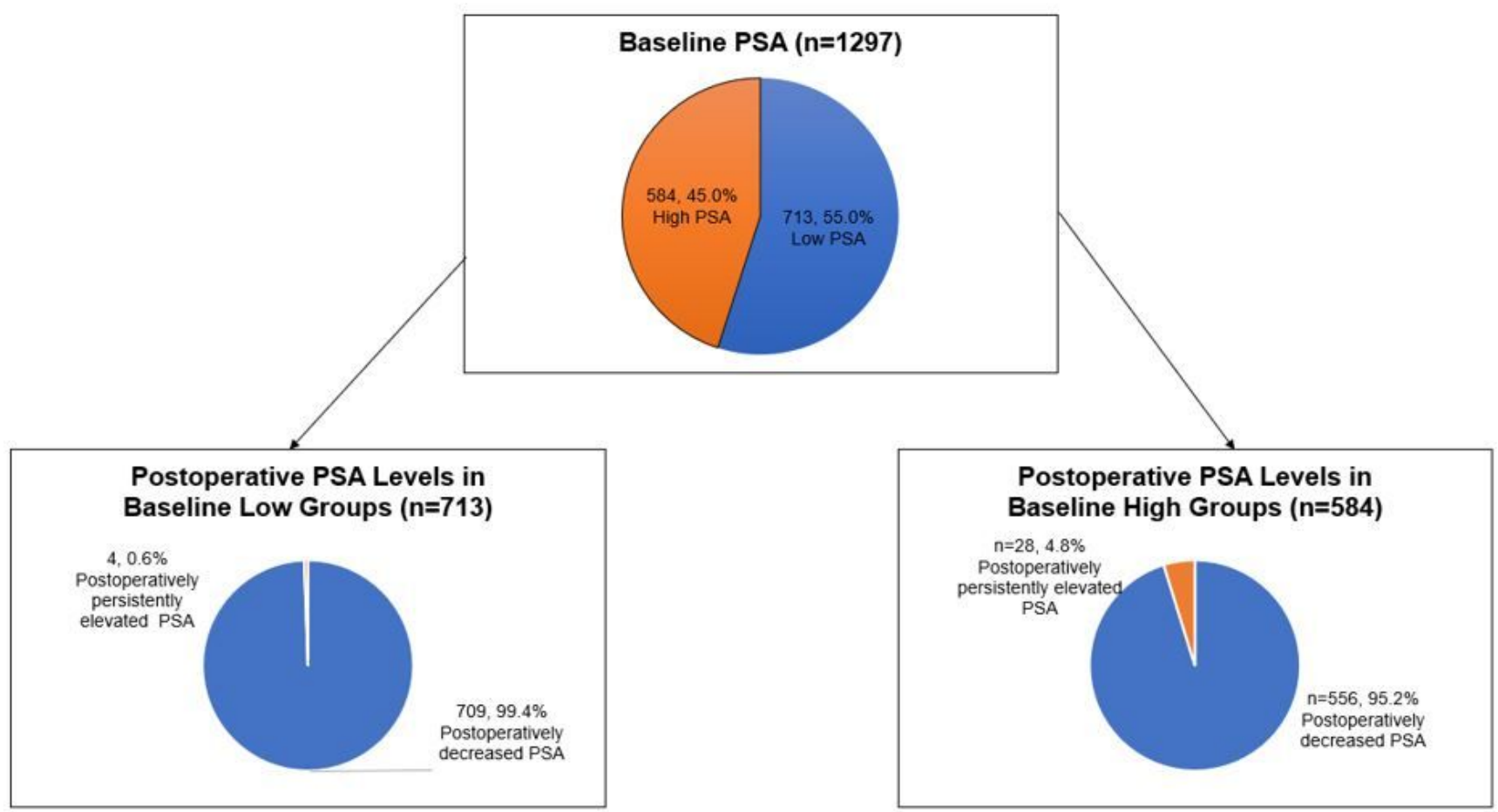

\section{Figure 1}

Natural history of PSA change according to baseline high and low PSA groups A. Changes in PSA from baseline low and high PSA groups at postoperative 6 months; the red lines indicate the patients in which PSA increased postoperatively. The patients whose PSA was persistently elevated were followed up and additional follow-up was conducted, the postoperative follow-up was usually discontinued at 6 months if there were no abnormalities revealed. The decrease rate of PSA was $66.7( \pm 23.6) \%$ in the total PSA group, and $54.3( \pm 23.9) \%$ in the low group, and $79.6( \pm 14.7) \%$ in the high group. B. Natural history of PSA change according to baseline high and low PSA groups. Among the patients who were 
preoperatively low ( $n=713), 709(99.4 \%)$ decreased and maintained low postoperatively, and among the patients who were high at baseline $(n=584), 556(95.2 \%)$ were persistently postoperatively decreased.

\section{Supplementary Files}

This is a list of supplementary files associated with this preprint. Click to download.

- supplementarymaterials.docx 